\title{
LA NUEVA OLA DE REGULACIÓN DE LOS GRUPOS DE PRESIÓN PARLAMENTARIOS ${ }^{1}$
}

\author{
Rafael RuBio NúÑEZ \\ Departamento de Derecho Constitucional \\ Facultad de Derecho \\ Universidad Complutense de Madrid \\ rafa.rubio@der.ucm.es
}

\begin{abstract}
RESUMEN
La regulación de los grupos de presión se ha extendido por todo el mundo en los últimos años. Esta regulación abarca no solo las actividades de influencia frente al poder ejecutivo, sino también ante el poder legislativo. Cuando se regulan de manera especifica estas actividades frente a las Cámaras, la normativa comparte una serie de elementos comunes con la regulación del ejecutivo y una serie de peculiaridades que se adaptan a la singularidad de las Cámaras. Estas ofrecen nuevas posibilidades para convertir el lobby en un instrumento que fomente la participación ciudadana, ajustándose a los principios básicos de la democracia.
\end{abstract}

Palabras clave: Transparencia, Grupos de presión, Grupos de interés, cabildeo, Parlamento, Parlamento Abierto, Participación, Información.

\section{ABSTRACT}

The new trend to rule lobbies at parliament has spread worldwide in the past few years. Such ruling not only covers major activities opposite executive powers but also opposite legislative powers. When such activities are specifically ruled opposite both the Houses, the law shares certain common ground with the laws of executive power and certain idiosyncracies which clearly adapt to the uniqueness of the Houses. Such offer new opportunities to turn the lobby into a tool to promote the citizen's participation, sticking to basic principles of democracy.

Keywords: Transparency, lobbies, interest groups, lobbying, Parliament, Open parliament, Participation, Information

${ }^{1}$ Este trabajo ha sido elaborado con el apoyo de los Proyectos I+D+I de Excelencia del MINECO, «El avance del Gobierno Abierto. Régimen jurídico constitucional de la implantación de políticas de transparencia, acceso a la información, datos abiertos, colaboración y participación especialmente a través de las TIC y del gobierno electrónico» DER2015-65810-P (2016-2018), e «Interacción entre representación y participación en la producción normativa» DER2015-68160-C3-3-P. Así como del Grupo de Investigación UCM sobre participación, tecnología y democracia. 


\section{ZUSSAMENFASSUNG}

Die gesetzliche Regulierung der parlamentarischen Interessengruppen hat sich in den letzten Jahren über die ganze Welt ausgedebnt. Diese Regulierung umfasst nicht nur die Aktivitäten der Einflussnabme gegenüber der Exekutive, sondern auch gegenüber der Legislative. Wenn diese Aktivitäten in spezifischer Weise gegenüber den Kammern reguliert werden, teilt die Gesetzgebung einige gemeinsame Elemente mit den Regelierungen der Exekutive und eine Reihe von Besonderheiten, die diese Aktivitäten spezifisch an die Kammern anpassen. Diese bieten neue Möglichkeiten, um die Lobbyarbeit zu einem Instrument der Bürgerbeteiligung werden zu lassen, das sich an die Basisprinzipien der Demokratie anpasst.

Schlüsselwörter: Transparenz, Interessengruppen, Lobby, Lobbyismus, Parlament, offenes Parlament, Beteiligung, Information.

SUMARIO: I. LA IMPORTANCIA DE LOS GRUPOS DE PRESIÓN EN EL PROCEDIMIENTO PARLAMENTARIO.-II. EL IMPULSO GLOBAL DE LA REGULACIÓN DEL LOBBY PARLAMENTARIO.-III. EL CONTENIDO DE LA REGULACIÓN DEL LOBBY PARLAMENTARIO.-1. Definición.-2. El registro.-3. Códigos de Conducta.-3.1. Lobistas.-3.2. Parlamentarios.-4. Medidas complementarias.-4.1. Transparencia.-4.2. Participación.

\section{LA IMPORTANCIA DE LOS GRUPOS DE PRESIÓN EN EL PROCEDIMIENTO PARLAMENTARIO}

El lobby parlamentario cuenta con una amplia tradición en España. Los testimonios escritos se remontan a las Cortes de Cádiz, donde los tablejeros o cortadores de carne no desaprovechaban ocasión de defender sus intereses. Como consta en el archivo del Congreso de los Diputados en la sesión del 11 de marzo de 1811, se hizo lectura de una petición de Sebastián José Rigal en que, «como apoderado de los tablajeros o cortadores de carnes de los reinos de Sevilla, Córdoba, Jaén, Granada, Valencia, Murcia, Aragón, Navarra, ambas Castillas, provincias de Extremadura, Mancha, Rioja, señorío de Vizcaya y de los demás de España, solicita que se expida la competente real cédula en que se declare que los cortadores están comprendidos en la de 18 de marzo de $1783 \gg^{2}$.

Actualmente, el núcleo del poder normativo en nuestro sistema constitucional está en el Gobierno, canalizado a través de los proyectos de ley, y en torno al 90 por 100 de las normas tienen su origen en el poder ejecuti-

${ }^{2}$ M. A. GonZalo, «De Cádiz a change.org», http://sesiondecontrol.com/actualidad/ nacional/de-cadiz-1812-a-change-org/. 
$v^{3}$. En el Parlamento, Congreso y Senado, solo se modifican entre el 10 y el 15 por 100, especialmente en casos de gobiernos con mayorías.

Como recientemente hemos analizado en profundidad la regulación del lobby ante el poder ejecutivo ${ }^{4}$, vamos a centrarnos en la regulación de las actividades realizadas para influir ante el poder legislativo, especialmente ahora que es difícil encontrar mayorías claras de apoyo a los gobiernos y el poder legislativo adquiere nuevo protagonismo, tanto como fuente de la iniciativa parlamentaria como por su capacidad de introducir elementos determinantes durante la tramitación parlamentaria e incluso rechazar un proyecto de ley. Por cuanto que esta pluralidad no es solo algo propio del Congreso de los Diputados, sino que se comienza a dar también en los Parlamentos de las Comunidades Autónomas, consideramos interesante ofrecer una perspectiva general de la regulación de los grupos de presión parlamentarios y de sus principios básicos.

No es de extrañar que, en consecuencia, hayan aumentado las propuestas de regulación del lobby parlamentario en la línea del informe del grupo Greco, que establece en sus recomendaciones para España $(2013$, ii) la aprobación de normas sobre la relación de los miembros del Parlamento con lobistas y otros actores que buscan influir en el proceso legislativo 5 .

El contenido de las mismas busca un acceso justo y equitativo de los distintos actores al proceso de decisión de las políticas públicas para que estas decisiones se correspondan con las demandas de la sociedad civil; la regulación debe insertarse en un marco más amplio en torno a la participación política y la transparencia, y ajustarse al marco constitucional y las

3 E. JimÉnez ApARIcio, «El procedimiento de elaboración de los anteproyectos de ley: la fase gubernamental», en A. MENÉNDEZ y A. PAU (dirs.), La proliferación legislativa: un desafio para el Estado de Derecho, Madrid, Civitas-Colegio Libre de Eméritos, 2004, pp. 315 y ss.

${ }^{4}$ R. Rubio, «La democratización de la actividad de los grupos de presión: la regulación más allá del registro», Teoría y Realidad Constitucional, núm. 40 (2017), pp. 399-430.

${ }_{5}$ Fourth Evaluation Round, Corruption prevention in respect of members of parliament, judges and prosecutors. Evaluation Report. Spain. Adopted by GRECO at its 62 nd Plenary Meeting (Strasbourg, 2-6 December 2013), disponible en bttps://rm.coe.int/16806ca048.

En el punto 51 señala: «In Spain, the main issue concerning contacts of MPs with third parties, relates not so much to lobbying firms (there are just a few), but to the influential role played by interest groups and professional organisations (associations, foundations and unions). In a system in which MPs are generally following party discipline when casting votes (see paragraph 20), the trend would be for lobbyists/interest groups to prefer channelling their influence through parliamentary groups rather than through individual MPs. In the GET's view, it is important that there is appropriate transparency on this type of dealings in order to protect the legislative process from improper influence or the mere appearance of so. Improved transparency in this regard can only contribute to boosting the image of and the trust in the Parliament, as well as the individual MPs». 
peculiaridades socioeconómicas del país. Para conseguirlo esta regulación se basa en la definición del lobista y su actividad de lobby, el establecimiento de un registro, y el establecimiento de códigos de conducta para extender esta cultura de integridad y transparencia entre los servidores públicos y lobistas.

Esta regulación del lobby suele enfrentar un problema que tiene que ver con su ámbito de aplicación. Junto a los intentos de regular el lobby en territorios supra y subnacionales nos encontramos con un buen número de sistemas políticos que, desde una interpretación estricta del principio de separación de poderes, optan por regular de manera diferenciada las actividades de influencia ante el poder ejecutivo y el poder legislativo.

Se trata de un enfoque que, si bien no es novedoso, es poco habitual en la doctrina, que suele centrar su atención en una perspectiva más general, como podemos ver en clásicos como el de Ramírez ${ }^{6}$ o García Pelayo ${ }^{7}$, o en obras más recientes como El lobby en España. ¿Asignatura pendiente?» ${ }^{8}$, el reciente estudio de Transparencia Internacional ${ }^{9}$ y dos completos estudios colectivos en los que se presta escasa atención al enfoque parlamentario ${ }^{10}$. Lo mismo ocurre con ciertas tesis doctorales recientes ${ }^{11}$ que centran su estudio en el poder ejecutivo.

Desde la perspectiva de la influencia de estos grupos en el poder legislativo podemos destacar el trabajo de Fisas ${ }^{12}$, el de Fernández Sánchez ${ }^{13}$, el de Ponce ${ }^{14}$ y mi propia contribución ${ }^{15}$. También, en este campo específico,

${ }^{6}$ M. Ramírez, Los grupos de presión en la Segunda República Española, Madrid, Tecnos, 1969.

7 M. García Pelayo, Constitución y grupos de presión en América Latina, México DF, Universidad Nacional Autónoma de México, 1977.

8 AAVV, El lobby en España. ¿Asignatura pendiente?, Madrid, Algón, 2014.

9 M. Villoria, Una evaluación del lobby en España: análisis y propuestas, Madrid, Transparencia Internacional, 2014.

10 J. Molins, L. Muñoz e I. Medina, Los grupos de interés en España. La influencia de los lobbies en la política española, Madrid, Tecnos, 2016, y M. Villoria, J. M. GimeNO y J. Tejedor, La corrupción en España. Ámbitos, causas y remedios jurídicos, Barcelona, Atelier, 2016.

${ }_{11}$ I. Hernández Aguado, El buen gobierno de la sanidad: las influencias debidas e indebidas en la formulación de políticas de salud, Madrid, Universidad Complutense, 2017, y J. M. Elguero, Lobby y grupos de presión: análisis regulatorio y estudio en el sector asegurador, Madrid, Universidad Complutense, 2017.

12 V. FISAs, El lobby feroz, Barcelona, Icaria, 1998.

13 J. L. Fernández SÁnChez, La influencia de los grupos de interés en el proceso legislativo socioeconómico: el caso del sector asegurador, Barcelona, PPU, 2012.

${ }^{14}$ J. PonCE, Negociación de normas y lobbies, Pamplona, Aranzadi, 2015.

15 R. Rubio, «Los grupos de presión en España, una revisión pendiente», Revista de las 
conviene destacar en el ámbito europeo las obras pioneras de Pelegrin ${ }^{16}$ y Xifra ${ }^{17}$. Además se han defendido distintas tesis doctorales que prestan atención al poder legislativo como la de Córdova ${ }^{18}$.

Consideramos interesante retomar el tema diecisiete años después, ya que en los últimos años la regulación del lobby ha entrado a formar parte de las respuestas para recuperar la confianza en la política en todo el mundo. Esta nueva ola regulatoria está introduciendo novedades que afectan a la acción de los grupos de presión ante el Parlamento, por lo que, en nuestra opinión, merece esta actualización.

\section{EL IMPULSO GLOBAL DE LA REGULACIÓN DEL LOBBY PARLAMENTARIO}

En otro lugar hemos explicado recientemente ${ }^{19}$ cómo la tendencia general de la regulación sobre los grupos de presión tiende a centrarse en la definición más o menos amplia de los mismos y el establecimiento de una serie de obligaciones de transparencia para los que se encuentran dentro de esta definición. Es cierto que en los últimos años se ha empezado a desarrollar una tendencia diferente que, en la línea de la definición de Transparencia Internacional, trata de centrarse en el qué (las actividades de influencia, independientemente de quién las desarrolle) y no tanto en el quién las lleva a cabo. De esta manera el lobby sería un verbo más que un sustantivo y se podría definir como «la acción sobre los distintos actores que participan en el proceso legislativo» (Transparencia Internacional), y su regulación estaría centrada en esta actividad, incluyendo a cualquiera que desarrollara la misma.

En el Parlamento esta actuación se puede concretar de maneras distintas con la introducción de una iniciativa legislativa, ya sea a través de una Iniciativa Legislativa Popular o una proposición de ley por parte de un grupo parlamentario; el inicio del procedimiento a través de la admisión a trámite o su rechazo a través de una enmienda a la totalidad, o la

Cortes Generales, núm. 55 (2002), pp. 165-188, e íD., Los grupos de presión, Madrid, Centro de Estudios Políticos y Constitucionales, 2003.

16 E. Pelegrin, El lobby en la Unión Europea, Madrid, ESIC, 1997.

17 J. XIFRA, Lobbying. Cómo influir eficazmente en las decisiones de las instituciones públicas, Barcelona, Gestión 2000, 1998.

${ }^{18}$ D. Cordova, La regulación de los lobbies en Latinoamérica, Madrid, UCM, 2016.

19 R. Rubio, «La democratización de la actividad de los grupos de presión: la regulación más allá del registro», Teoría y Realidad Constitucional, núm. 40 (2017), pp. 399-430. 
introducción o modificación del contenido de la misma a través de la introducción de enmiendas por parte de los grupos en las distintas fases del proceso.

La regulación del lobby parlamentario forma parte de la ola regulatoria de la última década, en la que se ha producido un incremento exponencial en la regulación del lobby en el nivel nacional ${ }^{20}$. Si a comienzos del siglo XXI solo cuatro países habían aprobado leyes sobre esta materia $^{21}$, desde 2005 en adelante otros diez han desarrollado legislación en este sentido ${ }^{22}$, grupo al que se ha unido también la Unión Europea ${ }^{23}$. Esta tendencia global, a la que se van uniendo otros países que también están discutiendo cómo regular la materia ${ }^{24}$, presenta una base común que podemos encontrar en las recomendaciones de la OCDE aprobadas en el año $2010^{25}$. La OCDE ha desarrollado una labor de impulso con la creación de un estándar internacional al que pueden acudir los países para regular el lobby, y así lo demuestra la similitud de las legislaciones aprobadas ${ }^{26}$.

Como parte de esta ola, la regulación del lobby parlamentario se ha ido extendiendo en distintos países. Por ejemplo, en Polonia la ley establece tres registros separados para los poderes legislativo y ejecutivo (basado en el Act on Lobbying in the Legislative Process 2006), distinguiendo entre el Parlamento y el Senado. También Israel regula específicamente la organización de los lobbies en el Knesset ${ }^{27}$. Francia tiene regu-

${ }^{20}$ OECD, Lobbyists, Governments and Public Trust, vol. 3, Implementing the OECD Principles for Transparency and Integrity in Lobbying, Paris, OECD Publishing, 2014.

${ }^{21}$ Estados Unidos (1946), Alemania (1951), Australia (1983) y Canadá (1989).

22 Polonia (2005), Hungría (2006), Israel (2008), Francia (2009), México (2010), Eslovenia (2010), Austria (2012), Italia (2012), Países Bajos (2012), Chile (2014) y Reino Unido (2014). Vid. R. RuBio NúÑEZ, «La actividad de los grupos de presión ante el poder ejecutivo: una respuesta jurídica más allá del Registro», UNED-Teoría y Realidad Constitucional, núm. 40 (2017), p. 405.

${ }^{23}$ En el año 2016 se ha planteado convertir el registro en obligatorio, disponible en http://eur-lex.europa.eu/resource.html? uri=cellar:8a8de191-8648-11e6-b076-01aa75ed71a1.0001.02/DOC_1Eformat=PDF (consultado el 8 de febrero de 2018).

${ }^{24}$ Otros Estados como Argentina, Colombia o México, que llevan debatiendo sobre la cuestión desde hace más de diez años. Vid. OECD, Lobbyists, Governments and Public Trust..., op. cit., p. 15.

${ }^{25}$ Recommendations of the Council on Principles of Transparency and Integrity in Lobbying, disponible en http://www.oecd.org/corruption/ethics/Lobbying-Brochure.pdf (consultado el 8 de febrero de 2018).

${ }^{26}$ Aun así la OCDE señala cómo solo un tercio de sus miembros han regulado la materia (14 de 35) y cómo en ocasiones estos textos son incompletos o de difícil aplicación, fruto de la coyuntura y sin una visión a largo plazo. Vid. OECD, Lobbyists, Governments and Public Trust..., op. cit., p. 35.

${ }^{27}$ Enmienda núm. 5 de la Ley del Knesset 2008. 
laciones y códigos de conducta separados que corresponden a las dos cámaras: el Senado y la Asamblea Nacional ${ }^{28}$. La regulación de los lobbies en el poder legislativo mexicano también distingue entre la Cámara de Diputados ${ }^{29}$ y el Senado ${ }^{30}$. En Holanda, las Reglas de Procedimiento del Tweede Kamer (2012) regulan el acceso de los lobbies al Parlamento, estableciendo un registro específico para ellos. En Eslovenia, la Ley sobre la Integridad y la Corrupción 2010 creó un registro de lobbies que implica a la Asamblea Nacional en las actividades de regulación. Por último, la ley vigente en Chile aprobada recientemente estipula que el Congreso Nacional también está sujeto a la ley ${ }^{31}$. Todas ellas, menos esta última, responden en gran medida a los principios de la OCDE ${ }^{32}$.

En la Unión Europea la historia de la regulación de los grupos de presión parlamentarios se remonta a 1992, cuando el informe Galle planteaba la necesidad de regular el lobby en las instituciones europeas, y planteaba ya la necesidad de un registro en el Parlamento Europeo y un código de conducta para los lobistas, así como la obligación de los miembros del Parlamento de actualizar su declaración de intereses financieros anualmente e introducir inmediatamente un registro de los intereses financieros del personal de los miembros ${ }^{33}$. Tras el establecimiento de un primer registro, exclusivo del Parlamento en 1996, en el año 2011 se estableció un registro común para la Comisión y el Parlamento Europeos, el Registro de Transparencia ${ }^{34}$ (que fue objeto de reforma en 2014), así como un código de conducta común para los lobistas en sus contactos con los miembros de las instituciones europeas ${ }^{35}$ y un «código de conducta de los diputados al Parlamento Europeo en materia de intereses económicos y conflictos de intereses», que regula los contactos de los parlamentarios con personas ajenas a la Eurocámara. Desde 2016 se encuentra en circulación una

\footnotetext{
28 Art. 25 de La loi $n .^{\circ} 2016-1691$ du 9 décembre 2016 relative à la transparence, à la lutte contre la corruption et à la modernisation de la vie économique (bttps://bit.ly/2rZMAcu).

29 Reglamento de la Cámara de Diputados, título VIII, capítulo III.

${ }^{30}$ Reglamento del Senado, título IX, capítulo IV.

${ }^{31}$ Ley núm. 20.730, que regula el lobby y las gestiones que representen intereses particulares ante las autoridades y funcionarios (art. 4.5).

${ }_{32}$ Principles for Transparency and Integrity in Lobbying, disponible en bttp://www.oecd. $\mathrm{org} /$ gov/ethics/oecdprinciplesfortransparencyandintegrityinlobbying. htm.

33 Art. 9.4 del Reglamento del Parlamento Europeo.

34 P7_TA (2011). Decisión del Parlamento Europeo de 11 de mayo de 2011, sobre la celebración de un acuerdo interinstitucional entre el Parlamento Europeo y la Comisión relativo a un registro de transparencia común [(2010/2291 (ACI)].

${ }_{35}$ Anexo al Reglamento del Parlamento Europeo (DOUE, C 165 E/70, de 11 de junio de 2013).
} 
nueva propuesta de reforma que añade al Consejo al registro único y establece el registro obligatorio.

En España es bien sabido que los primeros intentos de regulación del lobby parlamentario se remontan al año 1978, cuando a iniciativa de Manuel Fraga Iribarne se propone introducir la regulación del lobby en la Constitución Española, en ese momento en proceso de elaboración. La propuesta era la de incluir dentro del art. 77 (sobre el derecho de petición ante las Cámaras) una mención expresa para que las comisiones parlamentarias pudieran recibir a estos «grupos legítimos de intereses» en sesiones públicas y se estableciera un sistema de control y registro de los mismos por medio de una ley orgánica ${ }^{36}$. La iniciativa fue rechazada por aquellos que entendían, por un lado, que la constitucionalización de su existencia suponía una suerte de legitimación y, por otro, por aquellos que veían en la figura sombras de las figuras de democracia orgánica del franquismo. Desde entonces y durante mucho tiempo no se registraron iniciativas para la regulación de los grupos de presión parlamentarios. Habrá que esperar más de diez años, hasta la IV Legislatura (1989-1993), para encontrar una proposición de ley relativa al establecimiento por el Congreso de los Diputados de un registro público de grupos de interés y un código deontológico de los mismos, así como varias proposiciones no de ley en la misma línea ${ }^{37}$, e incluso la solicitud de creación de una ponencia en el seno de la Comisión de Reglamento para debatir el establecimiento de un registro público de grupos de interés y sus normas de funcionamiento.

La primera se presentó en 1990 cuando, en el contexto del escándalo Juan Guerra, el hermano del vicepresidente del Gobierno al que se acusaba de usar un despacho oficial y realizar servicios de influencia política, el Grupo Popular presentó una proposición no de ley para regular los «despachos que gestionan intereses particulares confluyentes con interés

${ }^{36}$ La propuesta planteaba añadir dos apartados más a este punto. Se añadían dos párrafos: «3. Las comisiones podrán recibir delegaciones de grupos legítimos de intereses, en sesiones que siempre tendrán carácter público. 4. Una ley orgánica establecerá un sistema de control y registro para los grupos de intereses que actúen de modo permanente» (BOCG, núm. 44, de 5 de enero de 1978, pp. 698 y 699).

37 Proposición de ley relativa al establecimiento por el Congreso de los Diputados de un registro público de grupos de interés y un código deontológico de los mismos (122/000151); proposición no de ley relativa al establecimiento por el Congreso de los Diputados de un registro público de grupos de interés y un código deontológico de los mismos (162/000238); proposición no de ley sobre la regulación de los grupos de interés (162/000244), y solicitud de creación de una ponencia en el seno de la Comisión de Reglamento para debatir el establecimiento de un registro público de grupos de interés y sus normas de funcionamiento $(154 / 000046)$. 
públicos». La proposición, aprobada por unanimidad, buscaba aumentar la transparencia y el control e instaba al Gobierno a regular la actividad de gestión y aproximación de los intereses económicos privados con los intereses públicos. En dicha proposición se insistía en la necesidad de proteger los principios de transparencia, control y objetividad, y sugería tanto un registro de actividades como la modificación del Reglamento del Congreso para abarcar la influencia ante el poder legislativo.

Pocos años más tarde, en 1993, a propuesta del CDS, se vuelve a instar al Gobierno para una regulación de los grupos de interés que incluya un registro público y un código de conducta. La proposición, que parte del reconocimiento de la contribución de estos grupos a la democracia desde el enriquecimiento de los principios de representación y participación, insiste en la necesidad de que su actuación se realice con transparencia y publicidad, para encuadrar sus pretensiones en el interés general ${ }^{38}$.

Pasarán otros diez años, hasta la IX Legislatura (2008-2011), para volver a encontrar iniciativas parlamentarias en este sentido, aunque ambas, presentadas al inicio de la legislatura, no hablan explícitamente de las Cortes Generales y terminan caducando ${ }^{39}$. La siguiente legislatura, la X (2011-2015), sería la más prolífica en propuestas de ese tipo, y así, junto a varias proposiciones no de ley sobre la creación de un registro de lobbies ${ }^{40}$ (que caducan o son rechazadas), encontramos dos proposiciones de reforma del Reglamento del Congreso sobre la regulación del lobby ${ }^{41}$ (una es

38 Inicialmente se presenta una proposición no de ley relativa al establecimiento por el Congreso de los Diputados de un registro público de grupos de interés y un código deontológico de los mismos (162/000238), que resulta retirada, y a continuación la proposición no de ley sobre la regulación de los grupos de interés (162/000244), que resulta aprobada con modificaciones. Al mismo tiempo se presenta una solicitud de creación de una ponencia en el seno de la Comisión de Reglamento para debatir el establecimiento de un registro público de grupos de interés y sus normas de funcionamiento (154/000046), que también es rechazada.

39 Proposición no de ley sobre creación de un registro de lobbies o grupos de intereses (162/000007) de ERC-ICV (2008) y la proposición no de ley sobre creación de un registro de lobbies o grupos de intereses (161/000001).

40 Proposición no de ley relativa a la creación de un registro general de intereses o lobbies (162/001103); proposición no de ley para la creación de un registro de lobbies o grupos de intereses (162/000148) del Grupo Parlamentario de IU, ICV-EUiA, CHA, La Izquierda Plural (2012), y proposición no de ley para la creación de un registro de lobbies o grupos de intereses (161/000168).

${ }^{41}$ Proposición de reforma del Reglamento del Congreso de los Diputados sobre regulación de la actividad de los grupos de interés o lobbies (410/000011) y proposición de reforma del Reglamento del Congreso de los Diputados sobre regulación de la actividad de los grupos de interés o lobbies (410/000010). 
retirada y otra caduca). En esa legislatura el debate sobre el tema adquirió protagonismo durante la discusión de la Ley 19/2013, de 9 de diciembre, de Transparencia, Acceso a la Información Pública y Buen Gobierno, especialmente después de que el presidente del Gobierno Mariano Rajoy, en el debate del Estado de la Nación de febrero de 2013, señalara la necesidad de una «regulación parlamentaria de las organizaciones de intereses (los llamados lobbies), con medidas que clarifiquen cuáles pueden ser sus actividades y cuáles deben ser sus límites» ${ }^{42}$. En la fase de enmiendas a dicha ley todas las fuerzas políticas salvo el Grupo Parlamentario Popular plantearon enmiendas en el sentido de regular la actividad de estos gru$\operatorname{pos}^{43}$, aunque, al no ser el Parlamento sujeto directo de la ley, ninguna de ellas se refería al lobby parlamentario.

También en la XI Legislatura (2015-2016), a pesar de la ausencia de gobierno, se presentó una proposición de reforma del Reglamento del Congreso ${ }^{44}$ que, dada la duración de la legislatura, terminó caducando.

A pesar de los intentos y las diferentes propuestas, hoy no existe una regulación nacional del lobby en nuestro país, tampoco para su actuación en el Parlamento, y no parece claro que vaya a existir en un corto periodo de tiempo ${ }^{45}$.

42 Debate del Estado de la Nación, Mariano Rajoy, 20 de febrero de 2013, disponible en http://www.cepc.gob.es/docs/default-source/default-document-library/discurso-integro. $p d f$ ? sfursn $=0$ (consultado el 3 de noviembre de 2017).

${ }_{43}$ El Grupo Mixto solicitaba «un registro de representantes de grupos de interés [...] y que estos elaboraran informes periódicos de sus actividades y financiación, así como de sus interlocutores públicos y sus contribuciones de campaña no dinerarias y pagos en eventos a entidades (30)».

La propuesta de ERC consistía en «solicitar al Gobierno español la presentación ante el Congreso de los Diputados de un proyecto de ley de regulación de los grupos de interés o lobbies [...]. En esta regulación se establecerá la creación de un registro de lobbies, la regulación de un código de conducta con fuerza legal, las medidas de transparencia, publicidad y control que deben regirlos, así como las oportunas sanciones en caso de incumplimiento» (77 y 178).

Izquierda Plural solicitaba un proyecto de ley de regulación de los grupos de interés o lobbies que, entre otras cuestiones, «regule la creación de un registro de lobbies, la regulación de mecanismos de control y transparencia de su actividad, así como la descripción de infracciones y su correspondiente régimen sancionador» (259).

UPYD hablaba de «registros en cada administración y código de conducta» (362).

$\mathrm{CiU}$ planteaba un registro general de intereses, siguiendo la normativa europea al respecto. El registro de grupos de interés dependerá de la Agencia Estatal de Transparencia, Evaluación de Políticas Públicas y la Calidad de los Servicios. Y Código de Conducta (438).

${ }^{44}$ Proposición de reforma del Reglamento del Congreso de los Diputados para la creación del registro de los grupos de interés o lobbies (410/000001).

${ }^{45}$ GRECO manifiesta sus dudas ante la posibilidad de regular esta materia durante la XII Legislatura (punto 20). Vid. Fourth Evaluation Round, Corruption prevention in respect of members of parliament, judges and prosecutors. Interim compliance Report. Spain. Adopted by 
Esta ausencia de regulación nacional del lobby ha provocado que en España las Comunidades Autónomas y algunas entidades locales hayan comenzado a regular la actividad de los grupos de presión ${ }^{46}$. Entre ellas algunas han incluido la regulación de los grupos de presión parlamentarios. Aragón ${ }^{47}$ incorpora a «(l)os diputados de Cortes de Aragón, los miembros de la Cámara de Cuentas, el Justicia y su lugarteniente, y los miembros del Consejo Consultivo», estableciendo en la misma ley la normativa aplicable tanto al poder ejecutivo como al legislativo. Por el contrario, otras regulaciones incorporan una referencia a la necesidad de replicar la normativa en el poder legislativo, en virtud de la autonomía de las Cortes. En Castilla-La Mancha, a través de la disposición adicional primera, que señala: «Corresponderá a los órganos competentes de la Cámara o de las respectivas instituciones establecer en su reglamento o normas de gobierno las medidas específicas necesarias para adaptar, de acuerdo con sus peculiaridades, su régimen y funcionamiento a los principios y obligaciones contenidas en la legislación básica mencionada y en esta ley» ${ }^{48}$. Sin embargo, y ello es lo más destacado a los efectos que aquí interesan, el apartado número 3 de la referida disposición adicional dispone expresamente que las Cortes de Castilla-La Mancha «crearán y regularán un registro de grupos de interés», a fin de garantizar los principios de buen gobierno previstos en la ley. En el caso del Parlamento de Cataluña, debido a la exigencia estatutaria de respeto a la autonomía parlamentaria ${ }^{49}$, la disposición adicional quinta provocó la reforma de su Reglamento que sí contempla el citado registro ${ }^{50}$. En la Comunidad Valenciana ${ }^{51}$ y la Comunidad de Madrid ${ }^{52}$ la ley también insta a sus Asambleas Legislativas a promover la adaptación de su Reglamento en línea con lo señalado por la ley.

GRECO at its 78Th Plenary Meeting (Strasbourg, 4-8 December 2017), disponible en https://rm. coe.int/fourth-evaluation-round-corruption-prevention-in-respect-of-members-of/1680779c4d.

${ }^{46}$ En otros países como Estados Unidos y Canadá ya existían este tipo de regulaciones regionales y en los últimos tiempos algunas regiones italianas se han unido a esta corriente regulatoria.

${ }^{47}$ Art. 3 de la Ley 5/2017, de 1 de junio, de Integridad y Ética Públicas de Aragón.

${ }^{48}$ Disposición adicional primera de la Ley 4/2016, de 15 de diciembre, de Transparencia y Buen Gobierno de Castilla-La Mancha.

49 Art. 58 del Estatuto de Autonomía de Cataluña.

${ }^{50}$ Capítulo III del título VI (arts. 216 a 218) del Reglamento del Parlamento de Cataluña.

${ }^{51}$ Ley 25/2018, de 10 de diciembre, de la Generalitat, reguladora de la actividad de los grupos de interés de la Comunitat Valenciana (LRAGICV).

${ }_{52}$ Ley 10/2019, de 10 de abril, de transparencia y de participación de la Comunidad de Madrid, título IV, capítulo II, arts. 65-71 y disposición adicional sexta. 


\section{EL CONTENIDO DE LA REGULACIÓN DEL LOBBY PARLAMENTARIO.}

La actuación de los grupos de presión parlamentarios requiere una respuesta jurídica que, en la mayoría de los casos, pasa por establecer un registro público y accesible a través de la página web institucional en el que deberán inscribirse todas aquellas entidades o personas que, por cuenta propia o a nombre de terceros, quieran establecer canales de comunicación con los miembros del poder legislativo con el fin de influir en el proceso de toma de decisiones. Este registro, acompañado del código de conducta, son el modelo elegido habitualmente para garantizar que la acción legítima de influir en el poder legislativo se realice de manera transparente y conforme a la ley.

\section{Definición}

El primer eje de cualquier regulación del lobby es la definición del sujeto obligado por la norma, los grupos de presión o lobbies. Al referirnos a los lobbies, lo estamos haciendo a una diversidad de empresas y organizaciones que actúan por cuenta propia o en representación de terceros, recibiendo o sin recibir remuneración de ningún tipo. De esta forma, al hablar de lobbies podemos estar refiriéndonos a organizaciones tan distintas como ONGs, think tanks, asociaciones, fundaciones, empresas, uniones o confederaciones sectoriales-regionales, grupos empresariales, agrupaciones ciudadanas e, incluso, hasta movimientos sociales; ninguna de ellas renuncia a adoptar estas técnicas en defensa de los intereses defendidos, lo que hace mucho más difícil afrontar su estudio y su regulación. La naturaleza del actor produce discrepancias normativas. Salvo algunas excepciones que prefieren referirse a grupos organizados, la inmensa mayoría coinciden en hablar de organizaciones y personas sea cual sea su forma o estatuto jurídico, e incluso hablan de plataformas, redes u otras formas de actividad colectiva sin personalidad jurídica.

En el caso de los grupos de presión parlamentarios, nuestra propuesta de definición sería la de «toda unión de individuos, autónoma y organizada, que lleva a cabo acciones para influir en el poder legislativo en defensa de unos intereses comunes» ${ }^{53}$. Estos grupos desarrollarían acciones de

53 R. Rubio Núñez, Los grupos de presión, Madrid, Centro de Estudios Políticos y Constitucionales, 2003. 
influencia directa como entrevistas o comparecencias, así como otro tipo de actividades indirectas que buscan simplemente estar informados de los procesos que se están desarrollando en el Parlamento, ofrecer información básica sobre el sector y las materias que se defienden, crear un clima de opinión pública favorable y aquellas que promueven la movilización social como forma de apoyar sus intereses o introducir un tema en la agenda política ${ }^{54}$. Esta gestión de la información adquiere múltiples formas que van desde la transmisión de información directa a través de análisis regulatorios, estudios e informes, las entrevistas personales, la comparecencia ante una comisión parlamentaria o un grupo de trabajo, hasta otras actividades como el seguimiento legislativo, la identificación de los parlamentarios que lideran un tema concreto, el contacto recurrente con diputados y asesores parlamentarios. A esto se podrían añadir otras vías que buscan influir en los parlamentarios a través de la opinión pública y los medios de comunicación ${ }^{55}$, el respaldo científico ${ }^{56}$, la sensibilización ${ }^{57}$, la movilización ciudadana, etc. Así como otras muchas actividades que buscan simplemente mantener viva la relación y el clima de confianza, predisponiendo la voluntad de los actores que participan en la toma de la decisión ${ }^{58}$.

\footnotetext{
54 J. M. BERRY, Lobbying for the People. The Political Behavior of Public Interest Groups, Princenton, Princenton University Press, 1977.

55 Aunque tradicionalmente las acciones de lobby preferían permanecer al margen de la opinión pública, hoy en la sociedad de la información la sociedad reclama a la política y a los políticos más transparencia, más legitimidad (A. GuTIÉRREz-RuBI, La política vigilada, Barcelona, UOC, 2011), y estas nuevas dinámicas de la política exigen que las actividades que buscan incidir en la vida política se desarrollen de cara a la opinión pública y cuenten con apoyo social. Los lobbies han entendido que la opinión pública puede convertirse, además, en la forma de llegada más directa a los decisores. De esta manera, puede servir para introducir una cuestión en la agenda política, lograr una reunión que no termina de producirse, fortalecer la legitimidad de sus propuestas o cambiar los términos de una negociación.

56 bttp://www.bbc.com/mundo/noticias-37378264.

57 En esta línea el lobby también entiende la necesidad de trabajar la sensibilización, el camino más largo, pero también el más seguro, para lograr incidencia pública promoviendo «un cambio en diferentes planos: en el de los conocimientos, en el de los valores y en el de las actitudes (en el pensar, en el sentir y en el actuar)» (INGENIERÍA SIN FronTERA, 2010: 75). Así lo han entendido organizaciones como Médicos del Mundo, que en su página web señala cómo para lograr sus objetivos «necesitamos sensibilizar a la sociedad». Para este fin, elementos como congresos, seminarios, exposiciones, libros, documentales y campañas de sensibilización, aunque no tengan un objetivo de lobby específico, suponen una tremenda ayuda al fomentar y crear, si tienen éxito, una cultura y unos comportamientos que favorecerán para la defensa de los objetivos perseguidos.

58 En este campo se incluirían actividades tan diversas como invitaciones, regalos, la financiación de campañas electorales, las incompatibilidades, la creación de expectativas laborales o las puertas giratorias.
} 
Tras esta definición amplia, en la que cada vez es más habitual definir como lobista a aquel que hace lobby, y no al revés, la regulación existente se debate entre limitarse a aquellos que pretenden influir e incluso a aquellos con «capacidad de influir» o hacer valer algún interés. Otros amplían el campo para abarcar a todos aquellos que quieran interactuar con los representantes y los gestores públicos. En esta línea se plantean también discrepancia en torno a la necesidad, o no, de la profesionalización, algo recogido por algunos ordenamientos, aunque la inmensa mayoría no requieren de esta.

\section{El registro}

Como hemos señalado, la regulación de los lobbies se centra tradicionalmente en un registro en el que deben inscribirse los sujetos establecidos. El registro es público, accesible (a través de medios electrónicos) y gratuito. Y su contenido incluye unos mínimos: a) una relación, ordenada por categorías, de personas, así como la sede de su organización; b) la información que deben suministrar en relación con las actividades que realizan, con su ámbito de interés y con sus fuentes financiación, así como los fondos públicos recibidos; c) un código de conducta común. A esto se añade, en algunos casos, el sistema de control y fiscalización, y los mecanismos de denuncia.

Entre los derechos que concede la inscripción se encuentran el actuar como lobby, pudiendo mantener reuniones en defensa de los intereses propios y ajenos ante los miembros de la administración, presentarse como tal haciendo constar su inscripción, hacer constar su contribución en las consultas públicas, ser informado sobre las mismas, así como sobre otro tipo de información acerca de la actividad formativa y de divulgación de su interés.

Junto al registro se suele establecer la presentación de informes periódicos que detallen los procedimientos normativos en los que se ha participado, quién ha participado en los mismos, los responsables públicos con los que se ha tenido relación durante el periodo, el presupuesto dedicado a esta actividad o el dinero facturado en compensación de los servicios prestados, si se ha trabajado para terceros.

En aquellos sistemas en los que existe regulación de la acción del lobby en el poder legislativo y el ejecutivo, incluyendo los distintos niveles de la administración, se opta entre la gestión centralizada a través de un registro único o por un registro exclusivo del Parlamento en el que se pueden esta- 
blecer mecanismos de intercambio de información, el reconocimiento recíproco de actuaciones y la interoperabilidad registral con otros registros ${ }^{59}$.

El registro puede ser obligatorio o voluntario, y en este caso puede venir acompañado de un conjunto de incentivos para aquellos que decidan registrarse. La tendencia actual es tratar de establecer la inscripción en el registro como obligatoria, aunque, dadas las dificultades de implementación de esta medida, todavía son muchas las regulaciones que optan por establecer la inscripción voluntaria que buscan incentivar vinculando al registro el disfrute de ciertos derechos que incorporan en la ley. Estos pueden ser, entre otros, el acceso al Parlamento para los inscritos; información sobre iniciativas y proyectos legislativos relacionada con sus ámbitos declarados de interés, o acceso a reuniones o conferencias informativas que puedan organizarse en sede parlamentaria.

Es importante destacar como esta obligatoriedad no puede impedir ni restringir el ejercicio de los derechos inherentes al mandato parlamentario, así como el ejercicio de los derechos individuales de participación política, acceso o petición.

El modelo de registro obligatorio requiere establecer una serie de excepciones que, cuando existen, suelen incluir entidades públicas o semipúblicas, así como a aquellos actores reconocidos constitucionalmente para el ejercicio de la participación: asociaciones de consumidores y usuarios, sindicatos y organizaciones empresariales. Además, algunos optan por excluir a aquellos que prestan asesoramiento jurídico para defender los intereses afectados por el procedimiento administrativo en actividades de conciliación o mediación, o para el ejercicio de derechos o iniciativas establecidos por el ordenamiento jurídico.

Junto a la obligatoriedad de la inscripción ya comentada, es habitual encontrar otro tipo de obligaciones de transparencia sobre las actividades que realizan, los terceros para los que trabajan y las retribuciones percibidas, así como de los gastos relacionados con su actividad, si existieran (esta información debe ser completa, correcta, actualizada y fidedigna, cuya publicidad aceptan); el compromiso de cumplir un código de conducta (que veremos a continuación), así como aceptar la aplicación del régimen de control y fiscalización y las medidas de sanción en caso de incumplimiento.

\footnotetext{
59 J. RidaO, Los grupos de presión, Valencia, Tirant lo Blanch, 2017.
} 


\section{Los códigos de conducta}

La regulación del lobby puede incluir códigos de conducta tanto para los parlamentarios como para aquellos que realizan actividades de presión, obligados a aceptar sus términos para realizar la inscripción.

\subsection{Lobistas}

El código de conducta es una de las obligaciones comunes a los inscritos en el registro. Su contenido incluye la identificación del declarante que lo suscribe, la entidad u organización a la que representa o por la que trabaja, y los intereses, objetivos o finalidades que persiguen; su compromiso de no obtener ni tratar de obtener la información o influir en la toma de decisiones de forma deshonesta, así como de proporcionar información actualizada y no engañosa tanto en el momento de la inscripción en el registro como posteriormente en sus actividades de influencia; aceptar que la información proporcionada se haga pública; el compromiso de no incitar, por ningún medio, a autoridades, cargos públicos, diputados o funcionarios a infringir la ley o las reglas de comportamiento establecidas por el código de conducta, y aceptar y cumplir las medidas adoptadas en caso de incumplimiento de las obligaciones establecidas por la ley o por el código de conducta.

Algunos añaden al código la obligación de informar a los cargos y autoridades con los que se relacionen que están actuando como lobby, así como la prohibición de difundir la información de carácter confidencial que conozcan en ejercicio de su actividad, así como el no ofrecer regalos, favores o servicios en condiciones ventajosas que puedan condicionar la neutralidad u objetividad de los funcionarios implicados, y el de colaborar con el órgano administrativo responsable del registro para llevar a cabo todas las actuaciones de control y fiscalización. Otros elementos menos habituales, pero que es posible encontrar en códigos de conducta, son la obligación de que el personal a su servicio no esté incurso en incompatibilidades y cumpla estrictamente la normativa aplicable sobre integridad y ética pública, así como el compromiso de respetar las instituciones y personas con quienes se relacionan en el desarrollo de su actividad; informar a las personas u organizaciones a quienes representen de la existencia de los principios de actuación y de las obligaciones que incluyen, y no represen- 
tar intereses contradictorios o adversos sin el consentimiento informado de las personas u organizaciones afectadas y no hacer uso abusivo del alta para darse publicidad, ni dar a entender que este les otorga una situación de privilegio ante los poderes públicos. Incluso existen códigos que incluyen la prohibición de vender a terceros copias de documentos obtenidos en su relación con los empleados públicos.

En algunos ordenamientos este código de conducta para los lobistas se plantea como un mínimo al que los declarantes pueden añadir un código específico del lobista o de la asociación profesional a la que pertenece, que deberá ser más exigente que el código general y que deberá inscribirse en el registro. En el caso de España existe un código de conducta de estas características, el de la Asociación de Profesionales de las Relaciones Institucionales $(\mathrm{APRI})^{60}$.

\subsection{Parlamentarios}

El comportamiento de los parlamentarios también puede ser objeto de regulación. En esta línea, el informe GRECO (2017) señala cómo la regulación del lobby tiene que ir más allá del registro de lobistas y considerar la parte del congresista y su conducta respecto a cualquier persona u organización que trata de persuadirle sobre sus obligaciones legislativas ${ }^{61}$.

De ahí que el código de conducta de los profesionales del lobby suela ir acompañado de un código de conducta de los parlamentarios. Este documento es una definición de los principios y reglas de comportamientos éticos que una organización entiende y asume que deben aplicarse a las actividades que desarrolla, tanto en sus relaciones internas como en sus relaciones externas, así como a sus actuaciones y a sus pautas de gobernanza. Aunque estos códigos suelen cubrir otros aspectos, por lo general afectan también a su relación con los grupos de presión.

La implantación de códigos de conducta en las Cámaras parlamentarias tiene su inicio en Estados Unidos y en otros países de la órbita anglo-

${ }^{60}$ http://relacionesinstitucionales.es/apri-asociacion-de-profesionales-de-relaciones-institu cionales/codigo-de-conducta/ (consultado el 6 de julio de 2018).

${ }^{61}$ Fourth Evaluation Round, Corruption prevention in respect of members of parliament, judges and prosecutors. Interim compliance Report. Spain. Adopted by GRECO at its 78Th Plenary Meeting (Strasbourg, 4-8 December 2017), disponible en https://rm.coe.int/fourthevaluation-round-corruption-prevention-in-respect-of-members-of/1680779c4d (consultado el 7 de febrero de 2018). 
sajona, pero se ha ido extendiendo a otros países $^{62}$, aunque han sufrido una evolución en la historia reciente del parlamentarismo, tratando de vencer la desconfianza creciente de la ciudadanía en la conducta de los parlamentarios y de las instituciones representativas en general. En este sentido, estos códigos de conducta parlamentarios se han centrado en cuestiones como la situación financiera de los representantes, su dedicación al trabajo como representante (ausentismo), el uso de información confidencial y los conflictos de intereses, o el uso indebido de las prerrogativas y prestaciones de que gozan los parlamentarios.

Trata de dar respuesta así a asuntos como los eventuales conflictos de intereses, en la que un «parlamentario toma una decisión o participa en ella en el ejercicio de sus funciones sabiendo que beneficiará de forma incorrecta y deshonesta a sus propios intereses financieros o a los intereses privados de otra persona, de forma directa o indirecta» ${ }^{63}$. Para dar respuesta a esto, los códigos de conducta establecen la obligación para los parlamentarios de presentar una declaración de bienes y/o de intereses. Estos códigos de conducta también suelen incluir las obligaciones que puede contraer el parlamentario frente a entidades a las que presta servicios, estableciendo un elenco de incompatibilidades y prohibiciones como, por ejemplo, concertar contratos con entidades exteriores para actuar en el Parlamento o en nombre de este.

Además de los aspectos financieros y los conflictos de interés, hay otros elementos como la asistencia a las sesiones plenarias y comisiones, obligando a motivar las ausencias y, en caso de no hacerlo, estableciendo sanciones como la publicación de la lista de ausencias, amonestacio-

${ }^{62}$ En 2005 se aprobaron en Canadá para la Cámara de los Comunes y para el Senado códigos de conducta que incorporaban sistemas de seguimiento que han dado resultados satisfactorios para la imagen de la institución y el control de los conflictos de interés. En 2011 el Parlamento Europeo aprobó un código de conducta que regula los intereses económicos y financieros de los parlamentarios. Ese mismo año, Francia aprobó un código deontológico para la Asamblea Nacional, además de unas leyes orgánicas (906 y 907) que regulaban los conflictos de interés de los parlamentarios y creaban la Alta Autoridad para la Transparencia en la Vida Pública. Como explica Gonzalo, el impulso reciente más importante para los códigos de conducta es la recomendación incluida en el Informe GRECO del Grupo de Países contra la Corrupción del Consejo de Europa, que incluye entre sus tres recomendaciones para los parlamentos precisamente la aprobación de estos documentos. Vid. M. A. GonzALO, «Conducta parlamentaria», disponible en bttp://sesiondecontrol. com/actualidad/nacional/codigos-de-conducta-para-parlamentarios/ (consultado el 7 de febrero de 2018).

${ }_{63}$ D. Beetham, El parlamento y la democracia en el siglo veintiuno: una guía de buenas prácticas, Ginebra, Unión Interparlamentaria, 2006, p. 102, disponible en bttp://wwwipu. org/PDF/publications/democracy_sp.pdf (consultado el 7 de febrero de 2018). 
nes, deducciones salariales, suspensiones temporales o incluso, de manera excepcional, el retiro del mandato parlamentario.

En el caso de España no hay duda que la elaboración y aprobación de un código de conducta de estas características, en la que deberían participar sus miembros, contribuiría a la apertura de nuestro Parlamento, tal y como recomendaba el Grupo GRECO (Grupo de Estados contra la Corrupción) del Consejo de Europa en sus recomendaciones tras la IV Ronda de Evaluación ${ }^{64}$.

\section{Medidas complementarias}

Junto al recurso al registro y los códigos de conducta, la regulación del lobby parlamentario debe añadir otros elementos complementarios que permitan una mayor transparencia de las actividades. Estas medidas complementarias ${ }^{65}$, además de asegurar la transparencia, deberían «asegurar un campo de juego equilibrado entre los intereses en juego [...] se precisa mayor garantía de participación para todos los afectados, más información acerca de los mecanismo de consulta y participación, y canales claros de comunicación entre ciudadanos y gobiernos/parlamentos» ${ }^{66}$.

\subsection{Transparencia}

En esta línea se plantean una serie de medidas como aumentar las herramientas de monitorización legislativa, empezando por mejorar la web de los Parlamentos y su portal de transparencia. El registro y los informes de actividades también pueden ser complementados con la agenda de los

\footnotetext{
${ }^{64}$ Fourth Evaluation Round, Corruption prevention in respect of members of parliament, judges and prosecutors. Evaluation Report. Spain. Adopted by GRECO at its $62 n d$ Plenary Meeting (Strasbourg, 2-6 December 2013), disponible en bttps://rm.coe.int/16806ca048 (consultado el 7 de febrero de 2018).

65 Propuestas elaboradas conjuntamente con Miguel Ángel Gonzalo y desarrollada con más detalle en M. A. GonZALO y R. RuBIO, «La transparencia parlamentaria en el Congreso de los Diputados» en A. Troncoso (dir.), Comentarios a la Ley 19/2013, de 9 de diciembre. Transparencia, acceso a la información pública y buen gobierno, Madrid, Civitas, 2017, pp. 283 y ss., e íD., «Vías de participación en el Parlamento. ¿Parlamentos abiertos?», en M. Pérez Moneo y J. Vintro, Participación política: deliberación y representación en las Comunidades Autónomas, Madrid, Congreso de los Diputados, 2017, pp. 145-179.

${ }^{66}$ «Una evaluación del lobby en España: análisis y propuestas», Madrid, Transparencia Internacional, 2014, p. 103
} 
parlamentarios. De esta manera se puede completar y contrastar la información del registro con la perspectiva del receptor de la influencia, incluyendo nuevos actores que no aparecen en el registro y la información utilizada en sus acercamientos ${ }^{67}$. A esto se añadiría la «huella legislativa» ${ }^{68}$ o memoria de análisis de trazabilidad, que incorporaría no solo una relación de los actores que han intervenido en los procesos legislativos, sino el momento en el que lo han hecho y la información proporcionada por cualquier vía durante el proceso.

Otro modo de contribución a la transparencia de las actuaciones de los grupos de presión en sede parlamentaria sería dotar de mayor apertura a las comparecencias, en comisión o subcomisión, así como a las comisiones de investigación y las ponencias de estudio ${ }^{69}$. Por un lado, aumentando su publicidad. Solo en caso de que lo solicite el compareciente, el contenido de la comparecencia tendrá carácter reservado (si bien debe ser pública la identidad del compareciente y si compareció a solicitud propia o de un grupo parlamentario determinado). Además, debería regularse el acceso, con las garantías que se estimen precisas, a la documentación de las comisiones de investigación o a los documentos parlamentarios que no son objeto de publicación oficial, como los informes de gobierno remitidos al amparo del art. 7 del Reglamento del Congreso de los Diputados o, igualmente, a los documentos entregados por los comparecientes en comisiones o ponencias de estudio.

También contribuye a la transparencia la publicidad de las peticiones ante las Cámaras a través de una plataforma que lo permita, en aquellos casos en los que los ciudadanos, en cumplimiento de la legislación de protección de datos, así lo autoricen. Asimismo, debe publicarse el trámite realizado incluyendo, en su caso, la respuesta recibida con el consentimiento previo del peticionario. Además, se podría publicar en la página web de las cámaras un informe semestral de las peticiones tramitadas

${ }^{67}$ Así lo recogen las recomendaciones del Informe GRECO en la Cuarta Ronda de Evaluación de España, dedicado a las medidas para combatir la corrupción entre los miembros del Parlamento y la judicatura, donde se señala la conveniencia de publicar las agendas de contactos de los parlamentarios con terceras partes, sobre todo en lo que se refiere a los agentes que pueden influir en el proceso legislativo. Vid. Fourth Evaluation Round, Corruption prevention in respect of members of parliament, judges and prosecutors. Evaluation Report. Spain. Adopted by GRECO at its 62nd Plenary Meeting (Strasbourg, 2-6 December 2013), disponible en bttps://rm.coe.int/16806ca048 (consultado el 7 de febrero de 2018).

${ }^{68}$ P6TA (2008). Resolución del Parlamento Europeo, de 8 de mayo de 2008, sobre el desarrollo del marco para las actividades de los grupos de interés en las instituciones europeas [2007/2114 (INI)].

${ }_{69}$ R. M. Rubio y A. Gonzalo, «La transparencia parlamentaria...», op. cit., p. 283. 
que recoja no solo la información numérica de las peticiones tramitadas, como se hace ahora en la web del Congreso, sino una información más detallada sobre el curso dado a las mismas, algo que hoy se hace ya en el Senado.

Existen otras vías, más indirectas, que pueden también contribuir a mejorar la transparencia de las actuaciones de los grupos de interés, como la colaboración con organizaciones de monitorización parlamentaria y organizaciones de la sociedad civil que desarrollan labores de control sobre el trabajo del poder legislativo ${ }^{70}$.

\subsection{Participación}

Existen además otras herramientas que pueden contribuir a mejorar la participación ciudadana y pueden ser utilizadas por los grupos de influencia. En primer lugar, el escaño ciudadano, que permite a los ciudadanos opinar sobre proyectos legislativos, en fase de tramitación, incorporando sus aportaciones al expediente ${ }^{71}$; las preguntas parlamentarias, que se convierten no solo en un mecanismo de control, sino también de información ${ }^{72}$, o la posibilidad de que cualquier persona física o representante de persona jurídica pueda registrar propuestas de enmienda al articulado de un proyecto de ley ${ }^{73}$ para su tramitación, siempre que sean asumidas por, al menos, un grupo parlamentario o agrupación parlamentaria, y presentadas como enmiendas al proyecto de ley en tramitación, haciendo constar en la motivación de las mismas su origen en la iniciativa ciudadana. Una figura original de participación que también puede servir a aquellos que

${ }^{70}$ Reglamento de las Cortes Valencianas (BOCV, núm. 328, 8 de abril de 2015), disponible en bttp://www.docv.gva.es/datos/2015/04/09/pdf/2015_2961.pdf. En su art. 3 se dice que: «Les Corts deberán establecer, en su caso, los correspondientes convenios de colaboración con las entidades que promuevan la democracia participativa por medio de las tecnologías de la información y la comunicación» (arts. 110 bis 3 y 110 ter 7).

${ }^{71}$ En España se ha puesto en funcionamiento en algunos Parlamentos autonómicos como el de Cataluña con el escaño 136 (art. 224.3 del Reglamento del Parlamento de Cataluña de 8 de julio de 2015, disponible en https://www.parlament.cat/document/cataleg/165485.pdf) o el escaño 110 en el Parlamento de Andalucía, las Cortes Valencianas (art. 110 bis 4) o las Cortes de Aragón (art. 305).

72 Tanto Murcia (art. 178 del Reglamento de la Asamblea de Murcia), como Canarias (art. 172 del Reglamento del Parlamento de Canarias), Andalucía (art. 165) y Aragón (266) admiten la presentación por parte de los ciudadanos de preguntas por escrito para su respuesta oral tanto en el registro como a través de la página web.

${ }^{73}$ Las Cortes Valencianas (art. 113 bis) y las Cortes de Aragón (art. 165). 
buscar influir en el Parlamento es la de las proposiciones no de ley de iniciativa ciudadana ${ }^{74}$.

También contribuiría a hacer más democrática esta participación permitir que las organizaciones cívicas o empresariales pudieran solicitar comparecer ${ }^{75}$, estableciendo un procedimiento de las comparecencias de particulares y entidades cívicas abierto y motivado, a través de un canal público en el que todos puedan conocer las solicitudes de comparecencias y su interés o relación con la materia que se encuentra en estudio o tramitación. La aceptación sería la regla general, salvo acuerdo contrario de una mayoría cualificada (evitando así tantas comparecencias «fraudulentas», pero evitando también que la mayoría pueda vetar las comparecencias de entidades no afines $)^{76}$, rigiendo el principio de igualdad de trato a los que soliciten audiencias sobre la misma materia.

Por otro lado, estaría la posibilidad de reforzar la institución de las peticiones ante las Cámaras, establecidas en el art. 77 de la Constitución, y que se puede ejercer de forma individual o colectiva dirigiéndose a las Cámaras para poner en conocimiento de estas ciertos hechos, reclamar una intervención o ambas cosas a la vez. La publicación de estas peticiones, que ya hemos visto antes, es otra vía de contribución a la transparencia de las acciones de influencia, pero, además, la tecnología permite realizar dichas peticiones y adherirse a las mismas a través de Internet. De hecho, vemos como, donde las instituciones no lo han facilitado, la sociedad civil ha organizado campañas e, incluso, plataformas que han buscado hacer más sencillo y eficaz el uso de las peticiones a los parlamentarios.

${ }_{74}$ Establecida, por ejemplo, en el Reglamento de las Cortes de Aragón (art. 270).

75 Así lo establecen las Corts Valencianas (art. 182 ter), que incorporan a los alcaldes de municipios de la Comunidad Valenciana. El Parlamento de Cataluña (art. 106) incorpora también un trámite de audiencia a lo largo del procedimiento legislativo en las ponencias o subcomisiones a «las entidades y las asociaciones más representativas de carácter social, económico, sindical, humanitario, cultural y educativo». Andalucía (art. 112.1) permite incluir entre los interesados a las Administraciones Públicas y admite excepcionalmente la comparecencia de personas a título individual (art. 112.2), algo que también sucede en Extremadura (art. 165). En Asturias [art. 67.1.f)], además, si las comparecencias las proponen dos grupos o una quinta parte de los miembros de la Comisión, no hará falta la aprobación por la Mesa o por la Comisión (art. 67.3 y 4), reconociendo un derecho a las minorías inexistente en otros reglamentos parlamentarios.

76 Además, se podría garantizar el papel de los grupos parlamentarios asegurando a cada grupo, al menos, una comparecencia por iniciativa legislativa; de manera que, al igual que lo establecido en el art. 67.4 del Reglamento del Parlamento de Asturias, cuando la comparecencia sea solicitada por dos grupos parlamentarios o un número suficientemente amplio de parlamentarios miembros de la Comisión no se requiera aprobación por la Mesa de la citada comparecencia. 
Desde el punto de vista de la participación, además de la posibilidad de asociarse a peticiones colectivas podría simplificarse el procedimiento de registro; acortar los plazos de tramitación de las peticiones en el seno de la comisión parlamentaria correspondiente; desarrollar la convocatoria en audiencia especial de los peticionarios, posibilidad ya utilizada en el Senado, aunque esporádicamente, y que podría realizarse, según la propuesta de Aranda, a través de la reconversión de «la Comisión de Peticiones en una verdadera Comisión de Participación Ciudadana» ${ }^{77}$, en la que incluso se podría establecer la obligatoriedad de la convocatoria para aquellos que reúnan un número determinado de apoyos a su petición. E incluso se podría ir más allá facilitando que el organismo receptor de las peticiones pueda utilizarlas para presentar las iniciativas parlamentarias que estime adecuadas; dar traslado del escrito a los grupos parlamentarios para la adopción por los mismos de estas medidas; acordar su remisión a la comisión parlamentaria o a otras instituciones interesadas como el Defensor del Pueblo, o el autonómico, el Gobierno nacional, autonómico o local, incluso al Ministerio Fiscal o los tribunales, como hace el Parlamento de Aragón.

En el Derecho comparado existen otras formas de participación de los grupos de interés en el Parlamento como los intergrupos. Estos son grupos informales de diputados y senadores pertenecientes a distintas tendencias políticas que en ocasiones están abiertos a personas ajenas a las propias instituciones que, en aquellos lugares en los que existen, con más o menos apoyo institucional, se convierten de hecho en un punto de contacto entre expertos, afectados, interesados y parlamentarios. Estas instituciones podrían servir de correa de transmisión de las opiniones y solicitudes de las organizaciones y particulares. Promueven la transversalidad tanto entre miembros de distintos grupos políticos como entre miembros de distintas Cámaras, otorgando a determinados temas visibilidad, identificando personas de referencia y aportando formatos de relación con la formalidad propia del reglamento parlamentario, evitando a su vez el secretismo que acompaña en ocasiones este tipo de reuniones informales. De ahí que, evitando cualquier confusión con los órganos oficiales de la institución, se pudiera dotar de visibilidad y operatividad a estos grupos, respetando su informalidad, pero estableciendo un reglamento interno ${ }^{78}$.

77 E. Aranda Álvarez, «El Reglamento del Congreso de los Diputados. Propuestas para su reforma», Revista de Estudios Políticos, núm. 175 (2017), pp. 17-65.

${ }_{78}$ Así se organiza, por ejemplo, en el Parlamento de Cataluña. Vid. http://www.parla- 
La evaluación del propio proceso de participación en el Parlamento, como establece el Parlamento de Cataluña ${ }^{79}$, también serviría sin duda a este propósito.

Esta serie de medidas dirigidas a aumentar la transparencia y la participación en el Parlamento contribuirían sin duda al control y el desarrollo del trabajo de los grupos de presión en el Parlamento, ajustando su comportamiento a los principios democráticos y en su gran mayoría requerirían la modificación de los respectivos reglamentos parlamentarios.

ment.cat/web/composicio/intergrups/informacio-intergrup?p_legislatura $=10 E_{p} \_t i p u s=I G E p_{-}$ codi $=506$ (consultado el 7 de febrero de 2018).

79 En el art. 226 se establece la obligación para el Parlamento de evaluar el impacto «que los procesos participativos tienen sobre la actividad parlamentaria» y para tal fin se establece el deber de elaborar y publicar (en el portal de transparencia) una memoria que refleje, al final de cada tramitación, la influencia que el proceso participativo ha tenido en la ley aprobada. 